\title{
Experimental investigation on shear fracture at high strain rates
}

\author{
Christian C. Roth $^{1,2}$ and Dirk Mohr ${ }^{1,2,3}$ \\ ${ }^{1}$ Solid Mechanics Laboratory (CNRS-UMR 7649), Department of Mechanics, École Polytechnique, Palaiseau, France \\ ${ }^{2}$ Impact and Crashworthiness Lab, Department of Mechanical Engineering, Massachusetts Institute of Technology, \\ Cambridge MA, USA \\ ${ }^{3}$ Department of Mechanical and Process Engineering, ETH Zurich, Switzerland
}

\begin{abstract}
Adiabatic shear banding is a well-understood failure mechanism of metals at high strain rates. In addition, recent research on the ductile fracture of metals has demonstrated that shear localization at the microscale is also an important precursor of fracture initiation at low strain rates. This talk presents a new shear fracture specimen which is used to conduct fracture experiments on advanced high strength steel sheets at strain rates of up to $1 / \mathrm{s}$ in a hydraulic testing machine and for strain rates of up to 2500/s in a Split Hopkinson Bar system. The experimental result for a $22 \mathrm{MnB} 5$ steel show a significant increase in ductility as a function of strain rate. Results from scanning electron microscopy are also shown to provide insight into the effect of the strain rate on the shear localization at the microscale.
\end{abstract}

\section{Introduction}

A new shear specimen is presented that is suitable for characterizing shear fracture in sheet metals at low, intermediate and high strain rates. Due to the good observability of the flat specimen surface, the strains to fracture can be directly measured through digital image correlation (DIC). Experiments at three different strain rates are performed and analyzed. The strain rates pursued include slow quasistatic, intermediate $(\sim 1 / \mathrm{s})$ and fast $(\sim 2500 / s)$ loading rates. To shed more light on the shear band formation, the specimens are examined post mortem by electron backscatter microscopy. The material examined is a $1.02 \mathrm{~mm}$ thick quench-hardenable manganese boron steel (22 MnB5).

\section{Shear specimen}

For in-plane shear experiments on flat specimens, fracture is very likely to commence from the free boundary edge of the gage section. Despite the overall shear loading, plane strain tension stress states often prevail near the free boundaries, which normally have lower strains to fracture than pure shear. As a result, fracture initiates prematurely from the boundaries and the shear fracture strains obtained from such experiments can often only be interpreted as a lower bound. To address this well-known challenge, many authors make use of a reduced gage section thickness, so that the strain will be much higher in the specimen center, increasing the likeliness of fracture initiation under shear.

Inspired by the work of Till and Hackl (2013), Roth and Mohr (2015a) presented an in-plane shear fracture specimen of uniform thickness which features two parallel gage sections. This was achieved through an FEA-based optimization of the outer contour of the shear gage sections, making use of the Hosford-Coulomb fracture initiation model. The final shape of the specimen is shown in Figs. 1 and 2.

\section{Experiments}

Figure 2 shows the "smiley" specimens as extracted from a $1 \mathrm{~mm}$ thick $22 \mathrm{MnB} 5$ sheet. To obtain good dimensional accuracy, wire electric-discharge machining (EDM) is used. To perform digital image correlation, a random pattern that can be tracked by the DIC software (Vic2D, Correlated Solutions) is applied to the specimen surface with an average speckle size of $15 \mu \mathrm{m}$.

\subsection{Setup for low and intermediate strain rates}

All low and intermediate strain rate experiments are carried out on a MTS hydraulic testing machine with a $10 \mathrm{kN}$ load cell. A digital camera AVT Pike F-505B/C with a $90 \mathrm{~mm} \mathrm{1:1} \mathrm{macro} \mathrm{lens} \mathrm{is} \mathrm{used} \mathrm{to} \mathrm{observe} \mathrm{the} \mathrm{overall}$ displacement of the specimen at the positions highlighted through solid dots in Fig. 2. It is positioned at a distance of approximately $500 \mathrm{~mm}$ from the specimen, so that a spatial resolution of approximately $19 \mu \mathrm{m} /$ pix is obtained. A second camera AVT Pike F-505B/C with a $105 \mathrm{~mm} \mathrm{1:1}$ macro lens is utilized to closely observe the two gage sections of the specimen. It is positioned perpendicularly to the specimen surface at a distance of approximately $20 \mathrm{~cm}$, allowing a high spatial resolution of $3.5 \mu \mathrm{m} / \mathrm{pix}$.

All slow speed experiments are carried out at a crosshead displacement of $0.4 \mathrm{~mm} / \mathrm{min}$. Images are taken at a frequency of $0.5 \mathrm{~Hz}$ in conjunction with the commercial software VicSnap (Correlated Solutions). For the tests at intermediate strain rates, corresponding to a crosshead displacement of $8.3 \mathrm{~mm} / \mathrm{s}$, a high speed camera (Vision Research, Phantom 7.3) with a $105 \mathrm{~mm} \mathrm{1:1} \mathrm{macro}$ lens is utilized. With a resolution of $800 \times 600$ pixels, the images for DIC are acquired at a frequency of $1,000 \mathrm{~Hz}$ 


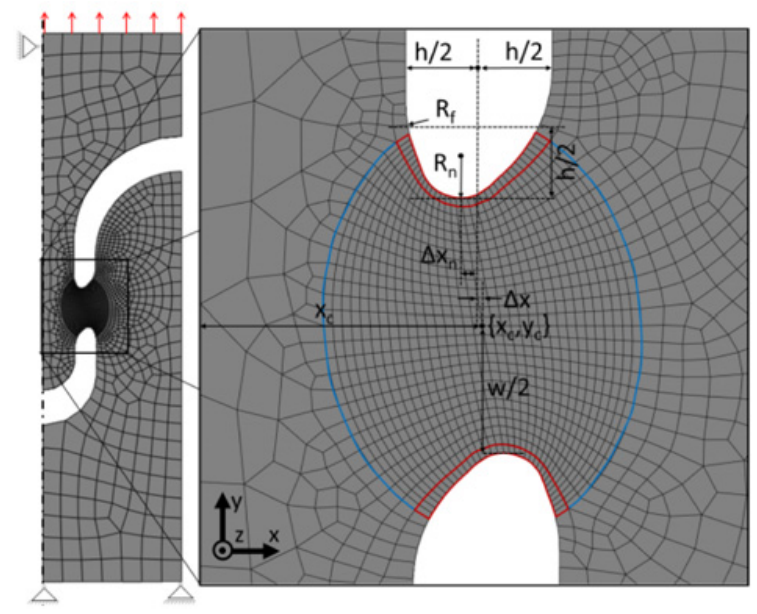

Figure 1. Finite element model of the optimized shear specimen geometry, including the parameterized variables.

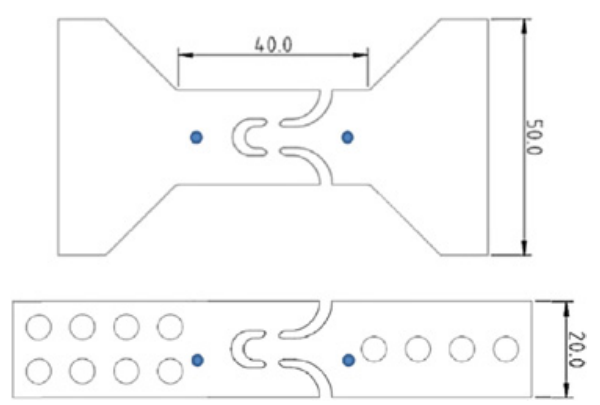

Figure 2. Specimens used for the experiments at low and intermediate strain rates (on the top), and high strain rates (on the bottom). Blue solid dots highlight the position of the virtual extensometer for relative displacement and speed measurements.

and a square pixel edge length of approximately $77 \mu \mathrm{m}$. The image acquisition is triggered by the rise in the force signal recorded by the load-cell.

\subsection{Setup for high strain rates}

A Split-Hopkinson Pressure Bar (SHPB) setup is used for the experiments at high strain rates in conjunction with a custom-made load-inversion device (Roth and Mohr, 2015b). The system comprises of a striker bar (3.80 $\mathrm{m}$ long, $20 \mathrm{~mm}$ diameter), an input bar (4.49 $\mathrm{m}$ long, $20 \mathrm{~mm}$ diameter) a load-inversion device and an output bar (4.35 $\mathrm{m}$ long, $20 \mathrm{~mm}$ diameter), with the latter positioned on top of the input bar. All bars are made of steel. In this configuration the setup allows for a valid experiment duration of $1.53 \mathrm{~ms}$. The geometry of the specimen used is shown in Fig. 2. As shown in Fig. 3, the loadinversion device consists of a pusher, which converts the compressive load from the input bar into a tensile load on the mounted specimen.

The opposite grip section of the specimen fits into a machined slit in the output bar, which is closed by four counter-sunk M5-12.9 screws, thus maintaining a symmetric mass distribution with respect to the output bar's center axis. The assembly is guided through bearings with lubricated contact surfaces. Strain gages positioned at a distance of $403 \mathrm{~mm}$ from the specimen/output bar
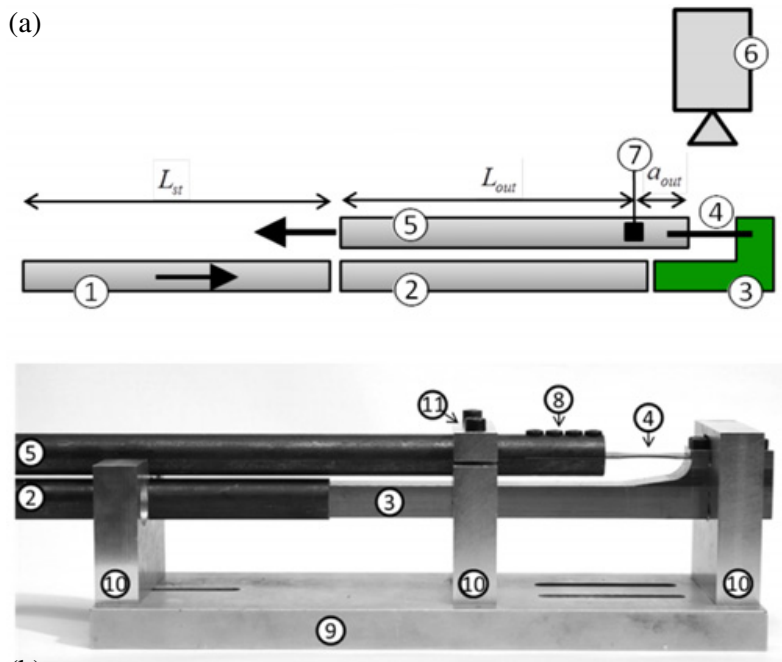

(b)

Figure 3. (a) Illustration of the experimental setup (b) Photograph of the Load Inversion Device - (1) striker bar, (2) input bar, (3) pusher of the LID, (4) specimen, (5) output bar, (6) high speed camera, (7) position of strain gauge, (8) grip section with slit, (9) base plate, (10) bearings, (11) removable clamp.

interface are used to record the output bar strain histories $\varepsilon_{\text {tra }}[\mathrm{t}]$. The history of the axial force $\mathrm{F}[\mathrm{t}]$ acting on the specimen is then calculated as

$$
F[t]=A_{\text {out }} E_{\text {out }} \varepsilon_{\text {tra }}[t]
$$

with $E_{\text {out }}$ denoting the Young's modulus, and $A_{\text {out }}$ the cross section area of the output bar. For the displacement measurements, the same high speed camera (Vision Research, Phantom 7.3) as for the experiments at intermediate speeds is employed. Two different setups are used: (i) To measure the global displacement history of the specimen, the camera is positioned at a distance of approximately $510 \mathrm{~mm}$ from the specimen, resulting in a spatial resolution of approximately $60 \mu \mathrm{m} /$ pixel. Images from an area of $576 \times 32$ pixels are obtained, which corresponds to approximately $35.1 \times 2.0 \mathrm{~mm}$ of the specimen surface. It is slightly offset from the center of the specimen to observe the onset of fracture. The camera is set to the highest possible acquisition frequency of $133 \mathrm{kHz}$, what corresponds to an image obtained every $7.5 \mu \mathrm{s}$. (ii) To measure the local displacement fields in the gage section of the specimen, the camera is positioned at a distance of approximately $250 \mathrm{~mm}$. Close-up images with a spatial resolution of $23 \mu \mathrm{m} /$ pixel are obtained from an area of $224 \times 72$ pixels at an acquisition speed of $133 \mathrm{kHz}$.

\subsection{Results}

It is observed that with increase in strain rate, the yield stress of the material increases as well - for the intermediate case the force-level is approximately $8 \%$ higher than for the slow one, while in the fast case it is approximately $25 \%$ higher at a displacement of $0.1 \mathrm{~mm}$. The shapes of the force-displacement curves for the slow and intermediate experiments are similar up to the force maximum; thereafter, the intermediate one 


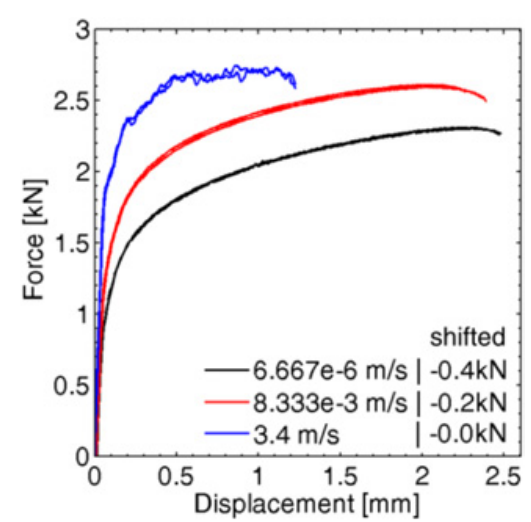

Figure 4. Plot of the force-displacement curves of the experiments (solid lines) and simulations (dots) at different strain rates and room temperature.

decreases slower than the slow ones towards fracture. The force-displacement curves of the experiments at high speed exhibit a different behavior. They show a plateau in the force level, before it decreases towards the onset of fracture. It is noteworthy that the displacement to fracture decreases with an increase in the loading speed.

A slow as well as one high strain rate experiment are examined with digital image correlation using the commercial software VIC2D (Correlated Solutions). Herein, the surface displacement is calculated by tracking the gray intensity distribution for a subset over subsequent images in conjunction with subset shape functions.

The error between the function and the image is minimized using a normalized squared differences approach algorithm. To obtain sub-pixel accuracy, the discrete greyscale distribution within a subset is interpolated by means of an 8-tap filter function. To assure comparability a step size of $0.1 \mathrm{~mm}$ is chosen. A resolution of approximately 28 pixel $/ 0.1 \mathrm{~mm}(\sim 3.5 \mu \mathrm{m} / \mathrm{pixel})$ is achieved for the slow experiments, while the high speed camera allowed obtaining only 4 pixel $/ 0.1 \mathrm{~mm}$ $(\sim 22.6 \mu \mathrm{m} / \mathrm{pixel})$ for the fast experiment. To improve the continuity of the displacement field and the correlation, the subset size is chosen to overlap by $1 / 8^{\text {th }}$. It is noteworthy that due to the limited acquisition frequency as well as the limited resolution of the high speed camera system, a minimum subset size of $9 \times 9$ pixel $(\sim 0.2 \times 0.2 \mathrm{~mm})$ had to be chosen for the fast case, while in the slow case a subset of approximately $0.1 \times 0.1 \mathrm{~mm}$ was utilized. No additional threshold options are chosen, while for the strain calculation with the Green-Lagrangian strain tensor a center weighted Gaussian filter smoothing over 5 data points is used

Figure 5 shows the evolution of the displacement field before the onset of fracture for the slow and the fast experiment. Clearly visible is the diagonal crack propagation which is seen as an indicator for shear fracture. The evolution of the shear strain along the white line in the displacement field is shown in the second row, while the third row depicts the evolution of the shear strain rate at the point where the shear strain has its maximum. It is observable that for both experiments the strain rate remains constant (approximately 0.005 for the slow case
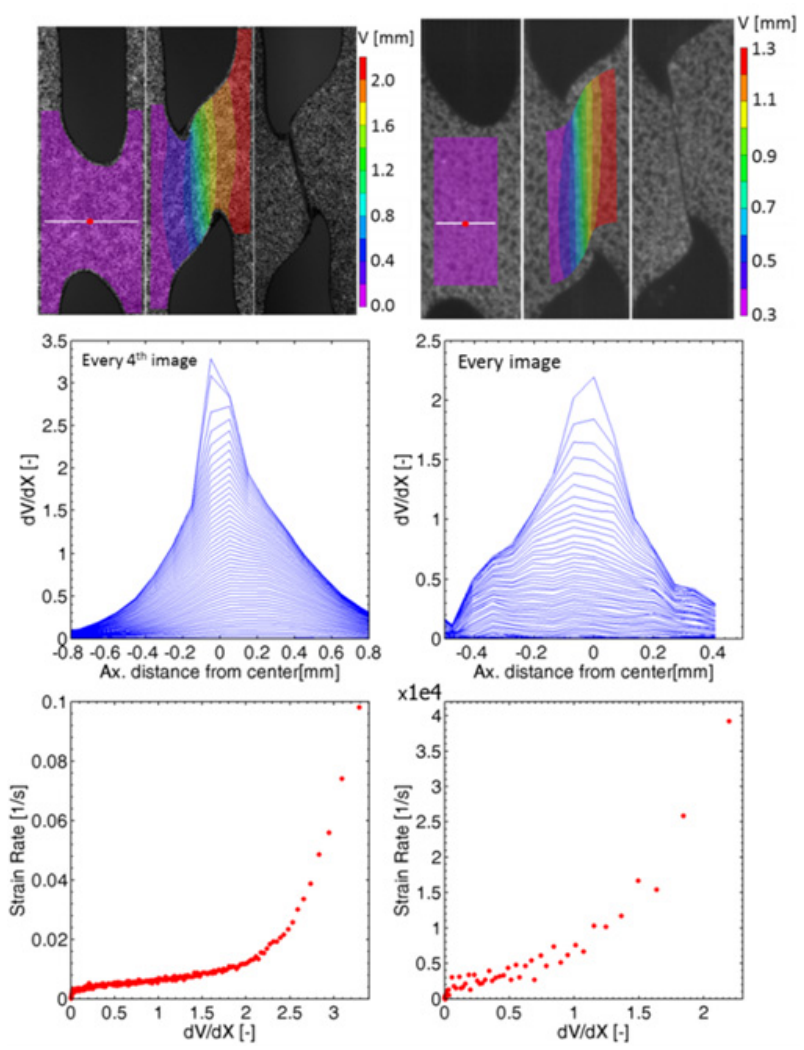

Figure 5. Displacement field and the extracted evolution of the strain along the white line and the strain rate at the location of the red dot (slow case left column, fast case right column).

and 2500 for the fast case), up to the point of localization. Here the strain rate begins to increase, reaching a value of an order of magnitude more at fracture. It has to be noted that the limited available resolution of the images for the high strain rate experiments, have a direct impact on the obtainable detail in the displacement measurement. Nevertheless, the location and width of the localization can be measured.

\section{Microscopic analysis}

One specimen each from the slow, intermediate and fast tests is examined closely using electron backscatter diffraction (EBSD) microscopy. The EBSD technique can be used to determine the crystallographic orientation of a material, thus effectively making orientation mapping and grain boundary identification possible. After extracting the zone of interest around the fractured gage sections with a diamond wire saw, the specimens are polished with $\mathrm{SiC}$ paper to grit $\# 4000$ and a $3 \mu \mathrm{m}$ diamond paste. Final polishing is performed by means of ion beams on a Gatan Pecs II ion polishing device. The specimens are exposed to the beam with an energy of $5 \mathrm{keV}$ stepwise from $6^{\circ}$ for $20 \mathrm{~min}, 4^{\circ}$ for $20 \mathrm{~min}$ and finally at a $2^{\circ}$ angle for 1 hour.

The observations are carried out on a scanning electron microscope FEI Quanta $600 \mathrm{~F}$ at $20 \mathrm{kV}$ and a spot size of 4.5. Using the HKL Channel software package, a $700 \mu \mathrm{m} \times 200 \mu \mathrm{m}$ wide area perpendicular to the rolling direction and close to the fracture plane is analyzed. Every data point is scanned three times and then averaged. 


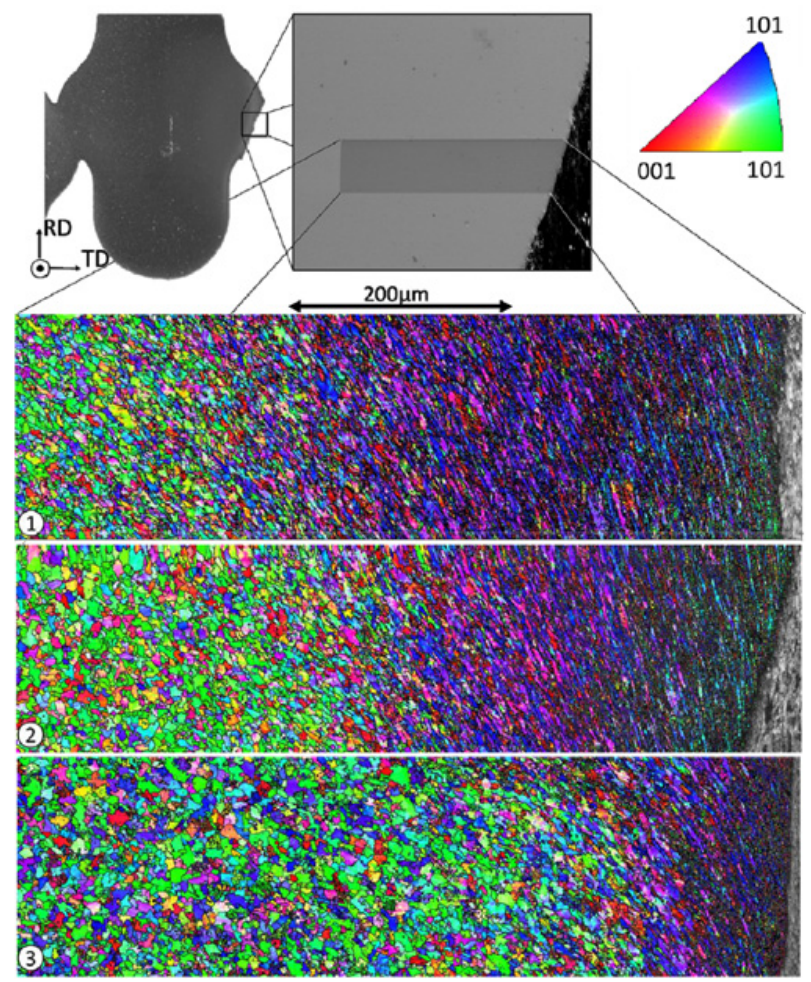

Figure 6. IPF-Y plots of the (1) slow, (2) intermediate and (3) fast experiments with added grain boundaries. Colors represent the grain orientation normal to the sample surface as defined by the stereographic triangle inset in the top right corner of the image.

With a step size of $0.43 \mu \mathrm{m}$ each image is obtained in around 16 hours and a resolution of approximately $1628 \times 465$ points per image is achieved. The data is postprocessed using very slight noise-reduction of the HKL Tango software (iteratively up to level 2 of 8).

\section{Conclusions}

A new flat shear fracture specimen is presented to perform experiments at low, intermediate and high strain rates. Its design is derived using an FEA based shape optimization methodology and allows for the direct observation of the surface strain field by means of DIC.

The experimental results show a positive strain rate hardening behavior of the material, while the fracture displacement decreases. Utilizing electron backscatter diffraction microscopy, it is shown that all experiments exhibit several deformed zones. It can be seen that with increasing strain rate the localization of deformation becomes more pronounced. In particular, the zone of localized deformation at low strain rates is about three times wider than that observed for high strain rates.

\section{References}

[1] D. Mohr, M. Dunand, and K.-H. Kim, Evaluation of associated and non-associated quadratic plasticity models for advanced high strength steel sheets under multi-axial loading. International Journal of Plasticity, (2010) 26(7): p. 939-956.

[2] C.C. Roth and D. Mohr, Effect of Strain Rate on Ductile Fracture Initiation in Advanced High Strength Steel Sheets: Experiments and Modeling. International Journal of Plasticity 56 (2014) 19-44.

[3] C.C. Roth, G. Gary and D. Mohr, Compact SHPB System for Intermediate and High Strain Rate Plasticity and Fracture, Experimental Mechanics (2015b) under review.

[4] C.C. Roth and D. Mohr, Basic Experiments on Sheet Metal with Propor-tional Loading Paths Until Fracture Initiation (2015a) to be submitted.

[5] R. Hill, A theory of the yielding and plastic flow of anisotropic metals. roceedings of the Royal Society of London. Series A, Mathematical and Physical Sciences, (1948) 193(1033): p. 281-297.

[6] T.B. Stoughton, A non-associated flow rule for sheet metal forming. International Journal of Plasticity, (2002) 18(5-6): p. 687-714.

[7] Till E., B. Hackl (2013). Calibration of plasticity and failure models for AHSS sheets, Proceedings of the International Deep Drawing Research Conference IDDRG 2013.

[8] G.R. Johnson and W.H. Cook. A Constitutive Model and Data for Metals Subjected to Large Strains, High Strain Rates and High Temperatures. in 7th International Symposium on Ballistics (1983). The Hague.

[9] G.R. Johnson and W.H. Cook, Fracture characteristics of three metals subjected to various strains, strain rates, temperatures and pressures. Engineering Fracture Mechanics (1985). 21: p. 31-48. 